University of Nebraska - Lincoln

DigitalCommons@University of Nebraska - Lincoln

Faculty Publications from the Department of Engineering Mechanics

Mechanical \& Materials Engineering,

Department of

2008

\title{
Computer simulation of radio frequency heating of model fruit immersed in water
}

\author{
Sohan Birla \\ University of Nebraska-Lincoln, sbirla2@unl.edu \\ Shaojin Wang \\ Washington State University, shaojin_wang@wsu.edu \\ Juming Tang \\ Washington State University, jtang@mail.wsu.edu
}

Follow this and additional works at: https://digitalcommons.unl.edu/engineeringmechanicsfacpub

Part of the Mechanical Engineering Commons

Birla, Sohan; Wang, Shaojin; and Tang, Juming, "Computer simulation of radio frequency heating of model fruit immersed in water" (2008). Faculty Publications from the Department of Engineering Mechanics. 82. https://digitalcommons.unl.edu/engineeringmechanicsfacpub/82

This Article is brought to you for free and open access by the Mechanical \& Materials Engineering, Department of at DigitalCommons@University of Nebraska - Lincoln. It has been accepted for inclusion in Faculty Publications from the Department of Engineering Mechanics by an authorized administrator of DigitalCommons@University of Nebraska - Lincoln. 


\title{
Computer simulation of radio frequency heating of model fruit immersed in water
}

\author{
S. L. Birla, S. Wang, and J. Tang \\ Department of Biological Systems Engineering, Washington State University, \\ 213 LJ Smith Hall, Pullman WA 99164-6120 USA \\ Corresponding author - J. Tang, tel 509-335-2140, fax 509-335-2722, email jtang@mail.wsu.edu
}

\begin{abstract}
The present study investigated the effect of different factors on temperature distribution within a spherical object in a parallel plate radio frequency $(\mathrm{RF})$ heating system. A finite element-computer simulation program - FEMLAB was used to solve the electromagnetic and Navier-Stoke equations for developing a model to study the effect of dielectric properties of a model fruit and its surrounding medium. The model fruit was prepared from $1 \%$ gellan gel for experimental validation of the simulation results. The results showed that spherically-shaped samples surrounded with air between RF electrodes and placed in the proximity of electrodes would not heat uniformly. Immersing the model fruit in water helped to reduce uneven heating within the model fruit, but created a new problem because the model fruits were found to heat unevenly at different horizontal positions. Horizontal and vertical model fruit positions with respect to electrodes significantly influenced the heating patterns inside the model fruit. The study suggested that movement and rotation of the spherical object is the only plausible solution for improving heating uniformity. The developed computer model can be further used for prediction of the heating pattern of fresh fruit as influenced by dielectric properties, size, shape, and surrounding media to design thermal treatments of specific commodities.
\end{abstract}

Keywords: dielectric properties, RF heating, heating pattern, quarantine, heating uniformity

\section{Introduction}

Radio frequency (RF) heating has been explored for rapid postharvest disinfestation of agricultural commodities (Frings, 1952; Nelson \& Payne, 1982; Wang, Tang, Johnson, Mitcham, Hansen, Cavalieri et al., 2002). A number of problems remain to be addressed before RF heat treatments can be successfully used in commercial applications. The most significant problem associated with RF heating is that heating is not uniform in fresh fruits (Tang, Ikediala, Wang, Hansen \& Cavalieri, 2000). Large temperature variations among and within fresh fruit reduce the effectiveness of a treatment and may cause severe thermal damage to its quality. In order to overcome non-uniform RF heating of fruits, Ikediala, Hansen, Tang, Drake \& Wang (2002) suggested a saline water immersion technique, but for large fruits such as apples and oranges uneven heating inside the fruit was still unacceptable. Birla, Wang, Tang \& Hallman (2004) showed improvement in heating uniformity in oranges and apples when the fruits were immersed in water and kept in motion by water jets during RF heating. However, even after rotating and moving fruits in the RF field, non-uniform heating was evident in some fruits. Computer simulation model could be an effective tool to examine the causes of non-uniform heating and in finding solutions to overcome this problem.

Neophytou and Metaxas (1996; 1997; 1998; 1999) started electromagnetic field modeling for industrial scale RF heating systems. Their work involved comparison of solution from both electrostatic and wave equations. They recommended that for small sized applicators, solution of Laplace equation is adequate, whereas for large size electrodes, wave equations should be used. Recently Chan, Tang \& Younce (2004) developed 


\section{Nomenclature}

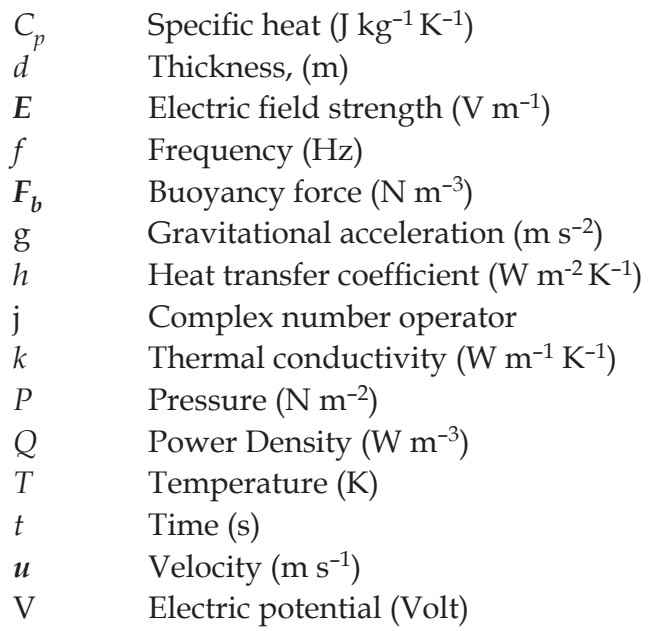

a simulation model for a RF parallel plate heating system, in which electromagnetic (EM) wave equations were solved using a finite element method. The above efforts were focused on modeling EM fields and predicting power density profiles. Yang, Zhao \& Wells (2003) added heat transfer module in modeling of RF heating of vegetable seeds packed in a rectangular box. They used the commercial software TLM-FOOD HEATING to solve the EM field by the transmission line method and the heat diffusion by the standard explicit finite difference time domain method. They reported discrepancies between simulated and experimental results, especially at the edges of the box. Veen, Goot, Vriezinga, Meester \& Boom (2004) derived a simplified expression for an EM field in very small spherical object immersed in an infinitely large surrounding medium. However, for large fruits such as oranges and apples, this expression is inappropriate. Marra, Lyng, Romano \& McKenna (2007) modeled RF heating of a cylindrical luncheon meat roll in a $600 \mathrm{~W}$ RF oven operated at $27.12 \mathrm{MHz}$ frequency. Their study successfully solved coupled EM and heat transfer equations using the commercial finite element method based software FEMLAB. Their study was focused on a small RF oven and the model did not include the effect of surrounding fluid media on the heating pattern which requires solving Navier-Stokes equations.

The overall goal of this study was to develop an effective simulation model to predict the transient temperature profile of a model fruit in RF treatments. Specific objectives were 1) to develop a computer model by solving coupled quasi-static EM field, and heat and momentum transfer equations using FEMLAB software, 2) to validate the predicted temperature distributions of a gel slab by experiments, and 3) to apply the validated model to better understand RF heating patterns in a model fruit as influenced by relative position, and surrounding media.

$\begin{array}{cl}\text { Greek symbols } & \\ \rho & \text { Density }\left(\mathrm{kg} \mathrm{m}^{3}\right) \\ \sigma & \text { Ionic conductivity }\left(\mathrm{S} \mathrm{m}^{-1}\right) \\ \varepsilon^{\prime} & \text { Dielectric constant } \\ \varepsilon^{\prime \prime} & \text { Dielectric loss factor } \\ \varepsilon_{\mathrm{o}} & \text { Permittivity of free space }\left(\mathrm{F} \mathrm{m}^{-1}\right) \\ \omega & \text { Angular frequency, }\left(\mathrm{rad} \mathrm{s}^{-1}\right) \\ \nabla & \text { Delta operator } \\ \beta & \text { Expansion coefficient }\left(\mathrm{K}^{-1}\right) \\ \mu & \text { Viscosity (Pa s ) } \\ \text { Subscripts } & \\ \text { air } & \text { Air } \\ \text { mat } & \text { Material } \\ \text { rms } & \text { Root mean square } \\ \text { w } & \text { Water }\end{array}$

\section{Materials and Methods}

\subsection{Physical model}

A $12 \mathrm{~kW}$ parallel plate RF heating system (operating at 27.12 MHz, Strayfield Fastran with E-200, Strayfield International Limited, Wokingham, UK) was used in this study. The RF heating system was consisted of a generator, an applicator and a metallic enclosure. The applicator had a pair of parallel plates in which the top electrode plate, with adjustable height, was inductively coupled to the tank oscillator circuit via feed strips. The dielectric material was sandwiched between two electrodes to form a working circuit (Wang, Ikediala, Tang, Hansen, Mitcham, Mao et al., 2001). RF heating involves EM interaction of the lossy material (dielectric material having considerable power dissipation capability) placed between the two plate electrodes. The absorbed RF power per unit volume $\left(Q, \mathrm{~W} \mathrm{~m}^{-3}\right)$ in the material is proportional to the square of the electric field strength $\left(E, \mathrm{~V} \mathrm{~m}^{-1}\right)$ and directly proportional to the dielectric loss factor $\left(\varepsilon^{\prime \prime}\right)$ and the frequency $(f, \mathrm{~Hz})$ (Choi \& Konrad, 1991):

$$
Q=2 \pi f \varepsilon_{0} \varepsilon^{\prime \prime} \boldsymbol{E}_{\mathrm{rms}}^{2}=\pi f \varepsilon_{0} \varepsilon^{\prime \prime}|\boldsymbol{E}|^{2}
$$

where $\varepsilon_{0}$ is the permittivity in free space $\left(8.85 \times 10^{-}\right.$ $\left.{ }^{12} \mathrm{~F} \mathrm{~m}^{-1}\right)$, and $E_{\mathrm{rms}}$ is the root mean square value of the electric field which is equal to $1 / \sqrt{2}$ times of the E-field amplitude.

Evaluation of the absorbed RF power density at any point inside the material requires the value of ' $E$ ' which is the function of the geometry and dielectric properties of the object, and electrode configuration (Marshall \& Metaxas, 1998). Moreover, dielectric properties are temperature dependent, hence estimation of $Q$ at any point requires simultaneous solving EM field and heat transfer equations. 
EM waves between 1 and $200 \mathrm{MHz}$ frequency fall within the radio band, and at $27 \mathrm{MHz}$ the wavelength is about $11 \mathrm{~m}$ in free space. In the present study, the electrode size $(1.05 \mathrm{~m} \times 0.80 \mathrm{~m})$ was very small compared with the wavelength of $27.12 \mathrm{MHz}$ used in our study. Therefore the primary mode of RF energy interaction was modeled as a quasi-static electrical field between two electrodes. Our approach was to systematically study the effect of the parameters that would significantly influence the heating patterns. Those parameters included the geometry of the object, relative location of the object in between the electrodes, and dielectric properties of the object and its surroundings.

\subsubsection{Governing equations}

The electric field at any point inside the electrodes is governed by Eq. (2) derived from a quasi-static approximation of Maxwell's equations (Choi \& Konrad, 1991):

$$
-\nabla \cdot\left(\left(\sigma+j 2 \pi f \varepsilon_{0} \varepsilon^{\prime}\right) \nabla V\right)=0
$$

where $j=\sqrt{-1}, \varepsilon^{\prime}$ is the dielectric constant, and $\sigma$ is the temperature-dependent conductivity $\left(\mathrm{S} \mathrm{m}^{-1}\right)$ and related to the dielectric loss factor as $\left(\sigma=2 \Pi f \varepsilon_{\mathrm{o}} \varepsilon^{\prime \prime}\right)$ in the RF range (Guan, Cheng, Wang \& Tang, 2004). The scalar voltage potential $(V, \mathrm{~V})$ is related to the electric field by $E=-\nabla V$, hence temperature dependent dielectric properties were sufficient to calculate the electric field strength at each point in the considered domain.

The geometry of most fruits such as cherries, apples, and oranges can be approximated as a sphere. Thermal diffusion in solid objects, for example fresh fruit, is governed by the Fourier transient transfer equation, but when immersed in fluid (water or air) it is governed by momentum transport and continuity equations (Zhang, Jackson \& Ungan, 2000):

Continuity equation $\nabla \cdot u$

Energy conservation equation $\frac{\partial T}{\partial t}+\boldsymbol{u} \cdot \nabla T=\frac{k}{\rho C_{p}} \nabla^{2} T+\frac{Q}{\rho C_{p}}(4)$

Momentum equation $\rho \frac{\partial \boldsymbol{u}}{\partial t}+\rho(\boldsymbol{u} \cdot \nabla) \boldsymbol{u}=-\nabla P+\mu \nabla^{2} \boldsymbol{u}+F_{b}$

where $C_{p}$ is the specific heat $\left(\mathrm{J} \mathrm{kg}^{-1} \mathrm{~K}^{-1}\right), k$ is the thermal conductivity $\left(\mathrm{W} \mathrm{m}^{-1} \mathrm{~K}^{-1}\right)$, $\mathrm{t}$ is the time $(\mathrm{s}), \mathrm{T}$ is the temperature $(\mathrm{K}), \rho$ is the density $\left(\mathrm{kg} \mathrm{m}^{-3}\right)$, and $\mu$ is the viscosity of the medium (Pa s). Similar to Eqs. (4) and (5) with the velocity component $u\left(\mathrm{~m} \mathrm{~s}^{-1}\right)$ in x-direction, the momentum and energy transport in $\mathrm{y}$ and $\mathrm{z}$ directions can be written with corresponding velocity components $v$ and $w$. The numerical solution of Eq. (5) requires pressure $(P$, $\mathrm{N} \mathrm{m}^{-2}$ ) to specify at least one point of the domain.

Buoyancy force $\left(F_{b}, \mathrm{~N} \mathrm{~m}^{-3}\right)$ in z-direction can be calculated from the Boussinesq approximation, whereby variations in density due to temperature are ignored but change in buoyancy force due to differential temperature is considered for the set up of a flow field.

$$
\boldsymbol{F}_{b}=\rho_{m} g \beta \Delta T
$$

where $g$ is the gravitational acceleration $\left(\mathrm{m} \mathrm{s}^{-2}\right), \beta$ is the volumetric thermal expansion coefficient $\left(\mathrm{K}^{-1}\right), \Delta T$ is the excess temperature from the reference one $(\mathrm{K})$, and $\rho_{m}$ is the fluid density at reference temperature $\left(\mathrm{kg} \mathrm{m}^{-3}\right)$. If the buoyancy force is the sole cause of motion, the convection is defined as free convection.

\subsubsection{Geometric model and boundary conditions}

It is impossible to take into account every details of the RF machine parts in the computation domain, as it demands excessive computer resources (Chan et al., 2004). One quarter of the RF electrode and the container was modeled to take advantage of the system symmetry. The upper electrode plate was drawn as an embedded element in 3-D to avoid a large number of mesh elements for a thin electrode plate. Symmetric surfaces were assigned thermal and electrical insulating boundary conditions as shown in Fig. 1.

Source electric potential (V) was applied to the upper electrode of the applicator and the bottom of the enclosure served as an electrical ground return $(V=0)$. An electrically shielding boundary condition was applied to the metallic enclosure walls such that $n \cdot(\sigma \nabla V)=0$ where $n$ is the unit vector normal to the surface of the wall. A thermal boundary condition of convective heat transfer was applied to the outer surfaces of the container (405 mm dia. $\times 127 \mathrm{~mm}$ height made of $6.25 \mathrm{~mm}$ thick polyethylene sheet). The water-air interface in the container was assigned to a convective heat transfer boundary condition. The convective heat transfer coefficient was assumed to be $20 \mathrm{~W} \mathrm{~m}^{-2} \mathrm{~K}^{-1}$ for water-air and container-air interfaces (Wang, Tang \& Cavalieri, 2001). At fruit-water and container wall-water interfaces no-slip boundaries were imposed in the momentum equations. At the water-air interface, the velocity in the normal direction $(w)$ and shear stress in the horizontal direction were assumed to be zero. Since solving Navier-Stokes equations needs to specify pressure at least at one point in the domain, atmospheric pressure was assigned at the top corner point of the container.

\subsubsection{Solution methodology}

Commercial finite element method based software FEMLAB (V3.2, COMSOL Multiphysics, Burlington, MA, USA) was used to solve the coupled electromagnetic, momentum and transport equations. Various steps involved in the modeling with FEMLAB software are outlined in Fig. 2. The computational scheme was to simultaneously solve the highly non-linear equations. An unstructured mesh consisting of Lagrange quadratic elements was created. To solve temperature and velocity values at the interfaces of spherical objects and surrounding media accurately, a relatively fine mesh was generated near the interfaces. A FEM solution was considered converging until the difference in maximum temperature between successive calculations was less 


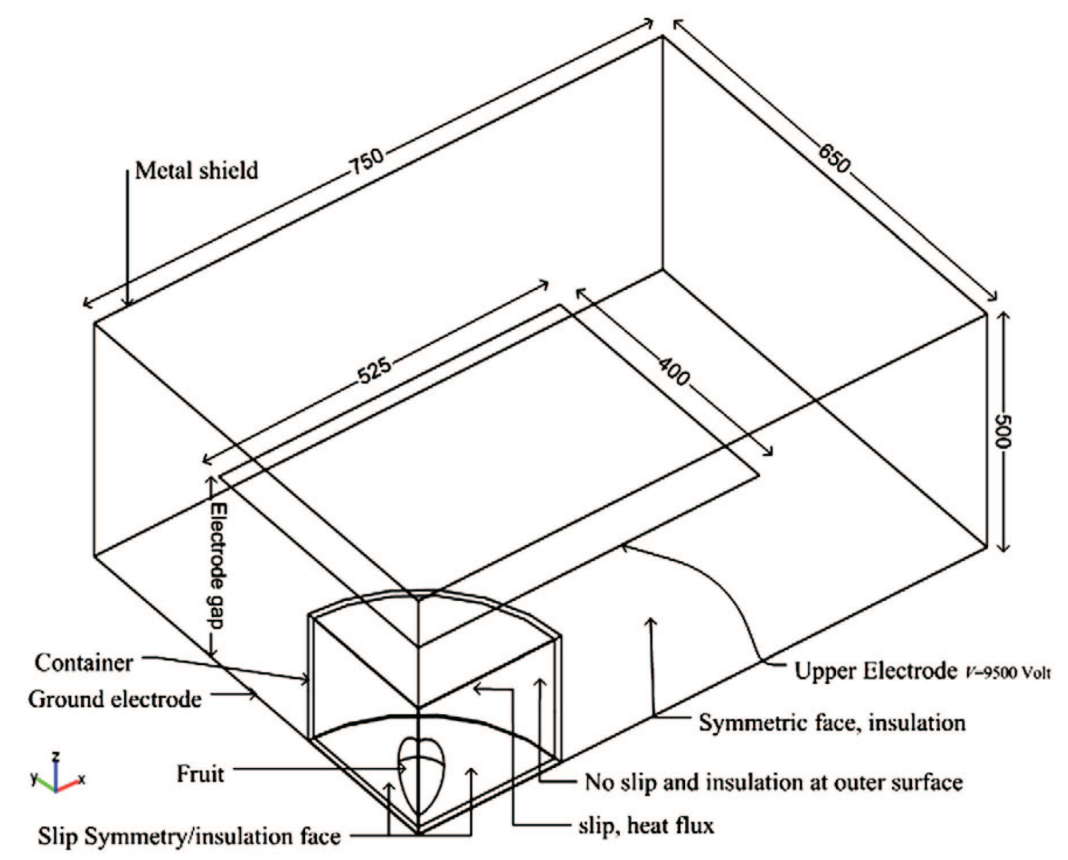

Fig. 1. Geometrical model of one quadrant of RF heating system (dimensions are in mm).

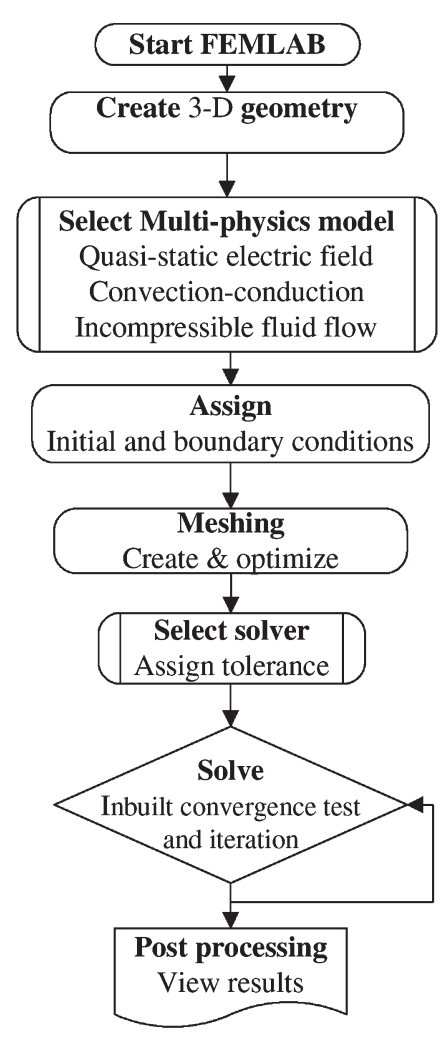

Fig. 2. FEMLAB modeling steps.

than $0.1 \%$ for a doubling of the number of elements. In the convergence studies, simulation results were found to be meshing independent when mesh sizes were reduced to $1 / 20^{\text {th }}$ of the maximum dimension of the domain. The optimum mesh generation yielded 3,641 nodes and 26,880 elements. The 'UMFPACK' differential equation solver in FEMLAB was used to achieve convergence. All computer simulations were performed on a Dell 670 work station with two each Dual-Core, $2.80 \mathrm{GHz}$ XEON processors and 12 GB RAM running a Windows XP 64bit operating system. Since the time-dependent equations are solved implicit, the accuracy is the only limiting factor for the time-step determination. One can specify the limits $(\mathrm{max} / \mathrm{min}$ ) for the time-step control for desired accuracy (COMSOL FEMLAB 3.3 User Guide, 2006). Simultaneous solutions of coupled transient equations took 12 $\mathrm{h}$ for a $10 \mathrm{~min} R \mathrm{R}$ heating process with $0.001 \mathrm{~s}$ initial and 1 s maximum time steps.

\subsubsection{Model input}

Modeling of the RF heating process requires knowledge of dielectric, thermal and physical properties of the load and surrounding medium. Density, thermal conductivity, and specific heat were assumed to be temperature-independent, whereas the dielectric properties of the materials were assumed to be temperature-dependent over the treatment temperatures range from 20 to $60^{\circ} \mathrm{C}$ for postharvest pest controls. Regression expressions obtained from the analysis of reported dielectric properties data were used in the model. Table 1 summarizes the properties of the various materials used in the simulations.

In the RF range, it is impractical to measure impressed voltage between two electrodes without distorting the electric field (Marshall \& Metaxas, 1998). Metaxas (1996) showed that for a typical industrial-scale system the voltage varies by only $7 \%$ between standby and full load. Therefore, a constant electric potential on the upper electrode is a realistic assumption. The analytical solution of Laplace equation for the electric potential between RF electrodes and heat dissipation in a slab 
Table 1. Electrical and physical properties of gel ball and water used in simulation

\begin{tabular}{|c|c|c|c|c|}
\hline Material proprieties & Gel ball & Tap water & Polypropylene & Air \\
\hline Thermal conductivity $\left(k, \mathrm{~W} \mathrm{~m}{ }^{-1} \mathrm{~K}^{-1}\right)^{\#}$ & 0.53 & 0.56 & 0.2 & 0.025 \\
\hline Density $\left(\rho, \mathrm{kg} \mathrm{m}^{-3}\right)^{\#}$ & 1010 & 1000 & 900 & 1.2 \\
\hline Specific heat $\left(C_{p^{\prime}} \mathrm{J} \mathrm{kg}^{-1} \mathrm{~K}^{-1}\right)^{\#}$ & 4160 & 4180 & 1800 & 1200 \\
\hline Exp. coefficient $\left(\beta, \mathrm{K}^{-1}\right)$ & $\mathrm{N} / \mathrm{A}$ & 0.0003 & $\mathrm{~N} / \mathrm{A}$ & 0.002 \\
\hline Dielectric constant $\left(\varepsilon^{\prime}\right)^{*}$ & $-0.21 \mathrm{~T}+86.76$ & $-0.48 \mathrm{~T}+84.74$ & $2.0^{\mathrm{v}}$ & 1 \\
\hline Loss factor $\left(\varepsilon^{\prime}\right)^{*}$ & $4.36 \mathrm{~T}+129.4$ & $0.33 \mathrm{~T}+11.1^{@}$ & $0.0023^{\mathrm{v}}$ & 0 \\
\hline
\end{tabular}

* (Wang et al., 2003a; Wang et al., 2003b) T - Temperature in ${ }^{\circ} \mathrm{C}$

\# (Rehman, 1995, pp. 179, 225)

(a) (Stogryn, 1971)

v (von Hippel, 1995, p. 327)

sandwiched between electrodes can be coupled together and an expression for electric potential on upper electrode can be obtained as (Metaxas, 1996):

$$
V=\left(d_{\text {air }} \sqrt{\left(\varepsilon^{\prime}\right)^{2}+\left(\varepsilon^{\prime \prime}\right)^{2}}+d_{\text {mat }}\right)\left(\sqrt{\frac{\rho C_{p}}{\pi f \varepsilon_{0} \varepsilon^{\prime \prime}} \frac{\mathrm{d} T}{\mathrm{~d} t}}\right)
$$

where $d_{\text {air }}$ and $d_{\text {mat }}$ are air gap and slab thickness (m), respectively.

The electric potential was estimated using the heating rate of a $1 \%$ gel slab $\left(405 \times 405 \times 47 \mathrm{~mm}^{3}\right)$ in the $12 \mathrm{~kW}$ RF unit. Procedure details are elaborated in the next section.

\subsection{Model validation}

\subsubsection{Model fruit preparation}

As a first step to validate the simulation model, it was more convenient to use a consistent homogonous spherical object. Thus, we developed a homogeneous model fruit made of $1 \%$ gellan gel. Wang, Tang, Cavalieri \& Davis (2003a) used gellan gel to prepare a model insect to study differential heating between insects and walnuts. One percent gellan gum dispersion (Kelcogel, Kelco Division of Merck and Co., San Diego, CA) in deionized water was prepared and the solution was heated to $90^{\circ} \mathrm{C}$ in $15 \mathrm{~min}$ (Tang, Tung \& Zeng, 1995). To be consistent with the dielectric properties of the most of fresh fruits $\left(\varepsilon^{\prime} \sim 84, \varepsilon^{\prime \prime} \sim 220\right.$ at $\left.20^{\circ} \mathrm{C}\right)$ as reported by (Wang, Tang, Johnson, Mitcham, Hansen, Hallman et al., 2003b), $0.17 \% \mathrm{CaCl}_{2}$ salt was added into the hot gellan gum solution. A polypropylene mould consisted of two hemispherical halves of $80 \mathrm{~mm}$ diameter was used to form the model fruit. The hot solution was poured into the mould though a hole in one half and allowed to cool at room temperature for $30 \mathrm{~min}$ to insure gel setting and easy removal of the ball (Tang, Tung \& Zeng, 1997). The dielectric properties of $1 \%$ gellan gel with $0.17 \%$ $\mathrm{CaCl}_{2}$ salt measured and reported by Wang et al. (2003a) over 20 to $60^{\circ} \mathrm{C}$ was used in the computer simulation.

\subsubsection{Experimental procedure for validation}

A $12 \mathrm{~kW}$ parallel plate RF heating system (operating at 27.12 MHz, Strayfield Fastran with E-200, Strayfield International Limited, Wokingham, UK) was used for experimental validation of the simulation results. The computer model was first validated with a slab load. A slab $\left(405 \times 405 \times 47 \mathrm{~mm}^{3}\right)$ made from $1 \%$ gellan gel spiked with $0.17 \% \mathrm{CaCl}_{2}$ was $\mathrm{RF}$ heated for $10 \mathrm{~min}$ with a $160 \mathrm{~mm}$ electrode gap to estimate input voltage on the upper electrode of the RF machine to be used for simulation purposes. The thermal image of the top surface of the gel slab was recorded using an infrared camera (ThermaCAM $^{\mathrm{TM}}$ Researcher 2001, accuracy $\pm 2^{\circ} \mathrm{C}$, FLIR Systems, Portland, OR, USA).

A simulation model prepared for a spherical object was validated with an experimental heating profile of the model fruit. The prepared model fruits (gellan gel ball) were allowed to equilibrate to room temperature (approximately $20^{\circ} \mathrm{C}$ ) prior to the experiment. To study the effect of the horizontal position of the fruit in the circular container, four model fruits were placed in the corners and one at the center of the container. The model fruits were secured at $25 \mathrm{~mm}$ above the container bottom. The container with the model fruits was placed at the center of the ground electrode. Ikediala et al. (2002) suggested immersion of fruit in saline water to minimize localized heating at contact surfaces. Therefore, in one experiment tap water (dielectric properties shown in Table 1) was poured into the container that completely immersed the gel balls.

Experimental heating patterns were obtained by varying the vertical position of the model fruits between electrodes with respect to the bottom electrode. The core temperature of centrally placed fruit was recorded and logged during the RF heating experiment using a fiber optic sensor (UMI-8, FISO Technology, Quebec, Canada). In additional experiments, five model fruits were RF heated in a fruit mover developed by Birla et al. (2004) to study the effect of rotation and movement of fruits during RF heating. The fruit mover had twelve numbers of water spray nozzles mounted on the periphery of a square container to rotate fruits on their axes and in a circular path. Details of the fruit mover can be found in our earlier publication (Birla et al., 2004). The RF heated model fruit was immediately bisected vertically and a thermal image was recorded with the 


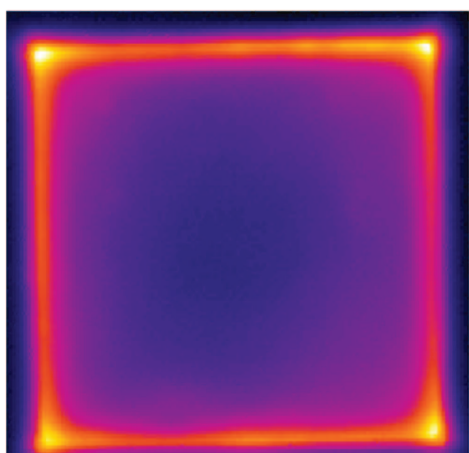

(a) Experimental

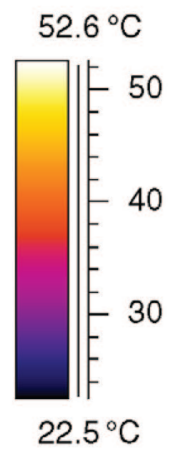

$22.5^{\circ} \mathrm{C}$

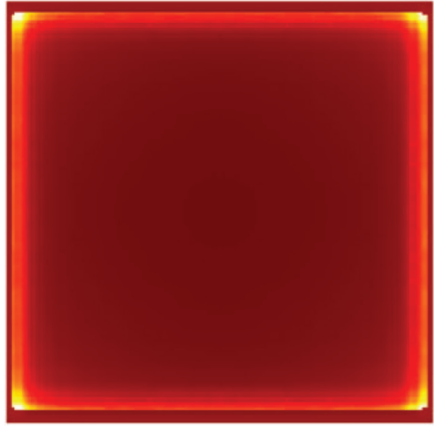

(b) Simulated $52.6^{\circ} \mathrm{C}$

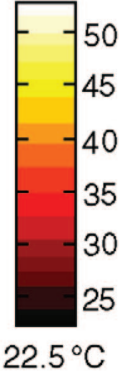

$22.5^{\circ} \mathrm{C}$

Fig. 3. Comparison of experimental and simulated surface temperature distribution of $1 \%$ gel slab $\left(405 \times 405 \times 47 \mathrm{~mm} \mathrm{~m}^{3} \mathrm{with} 20^{\circ} \mathrm{C}\right.$ initial slab temperature) subjected to $10 \mathrm{~min}$ of RF heating in a $160 \mathrm{~mm}$ electrode gap.

infrared camera for one of the cut surfaces within $10 \mathrm{~s}$ to avoid the surface cooling.

Simulation and experimental temperature data at core of the model fruit were subjected to statistical analysis. A parameter defined as root mean square (RMS) for temperature differences between simulation and experiment was calculated to validate the simulation results.

\subsection{Modeling of fruit rotation}

It has been demonstrated experimentally that rotation and movement of fruit during RF heating improves the heating uniformity (Birla et al., 2004). However, simulation of RF heating of rotating and moving object is very difficult because of the associated moving boundary conditions. To simplify simulation for this study, spherical objects were assumed to rotate on their own axes and each spot on any particular orbit was equally exposed to RF energy. The spherical object was further assumed to be composed of six concentric layers and RF energy absorbed on a particular layer to be uniform because of the 3-D rotation. This assumption was based on a concentric temperature contour over the fruit cross section observed with thermal images (Birla, Wang \& Tang, 2006). In computer simulation, power density was integrated over the individual layer and the total power density was divided by volume of the layer to estimate an average power density over the individual layer.

\section{Results and Discussion}

\subsection{Estimation of electric potential}

Fig. 3a shows the experimental temperature profile of the gel slab subjected to $10 \mathrm{~min}$ RF heating in a 160 $\mathrm{mm}$ electrode gap. Using the average slab temperature rise $\left(12.7^{\circ} \mathrm{C}\right)$ and dielectric properties of the gel at $25^{\circ} \mathrm{C}$ in Eq. (7), the input voltage was estimated to be 8,162 V. Since Eq. (7) is valid for an infinitely large slab and electrode dimensions (based on the assumption of no fringing field), the estimated voltage was not the actual value. In simulation, the estimated value of $V$ was used as a starting value and various input voltage values were tried for predicting the realistic transient temperature. A criterion for appropriate input voltage was based on $0.5^{\circ} \mathrm{C}$ RMS MS difference from measured temperature at the center of the gel slab. As a result of the simulations with a range of voltage, $9,500 \mathrm{~V}$ on the upper plate was selected for all further simulations. The simulated temperature distribution of the gel slab was in good agreement with the experimental one both in pattern and absolute temperatures (Fig. 3b). Marshall and Metaxas (1998) used a similar approach to estimate an appropriate voltage in modeling RF-assisted heating of particulates. Upon validation of the FEMLAB model for the slab, the model was further used to characterize the RF heating pattern in different geometries as influenced by the dielectric properties of medium and model fruit as well as locations of the model fruit relative to electrodes.

\subsection{Electric field pattern in various object geometry}

As the gel slab overheats at edges and corners of the slab because of fringe field effects, several simulation models were developed to better understand the effect of object shape on electric field distribution in the object. Fig. 4 shows the E-field distribution on the vertical cross section of sphere (80 $\mathrm{mm}$ dia), cylinder $(80 \mathrm{~mm}$ dia. $\times$ $80 \mathrm{~mm}$ height), and cube $\left(80 \times 80 \times 80 \mathrm{~mm}^{3}\right)$ immersed in water and situated in the center of the container with $25 \mathrm{~mm}$ above the bottom of the container. Under similar conditions, very high electric field concentrations occurred at the corners and edges of the cube, edges and the middle of the cylinder, whereas maximum E-field was at the bottom of the sphere. Interestingly, lowest electric field spot was always at the top central portion of all the objects. The spherical object is expected to heat more evenly as the electric field variation was only 37 $\%$ in sphere compared to $151 \%$ and $173 \%$ variations in the cylindrical and the cubical objects respectively. The problem of uneven heating can be further aggravated by the dielectric properties' dependency on temperature which may result in thermal runaway heating. Therefore, it is very important to devise means to eliminate these hot spots in the object. 

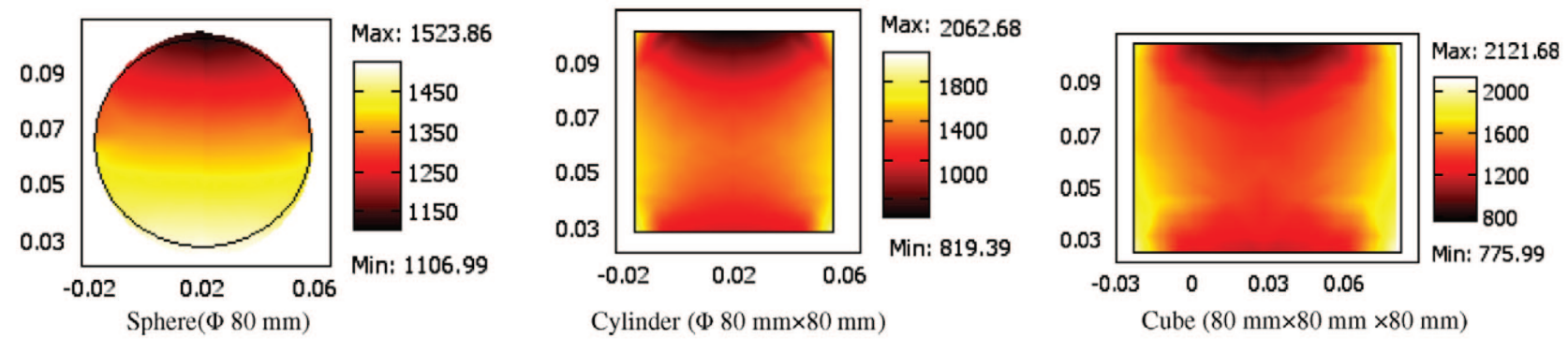

Fig. 4. Simulated electric field $(\mathrm{V} / \mathrm{m})$ distribution in the vertical cross sections of sphere, cylinder and cube made of gellan gel $(\varepsilon$ $=84-\mathrm{j} 220)$ placed in $195 \mathrm{~mm}$ RF electrodes and the objects were $25 \mathrm{~mm}$ above the bottom of the water $(\varepsilon=76-\mathrm{j} 18)$ filled container (405 mm dia $\times 127 \mathrm{~mm}$ height).

\subsection{RF heating of model fruit in air}

We learned from the results presented in the previous section that spherical objects should have higher electric field concentration at the fruit section close to the electrode. Fig. 5a corroborates the result as the maximum heating occurred at lower segment of the model fruit, placed at $25 \mathrm{~mm}$ above the ground electrode or 25 $\mathrm{mm}$ below the upper electrode in $160 \mathrm{~mm}$ of air gap between two RF electrodes. Experimental temperature profile over the fruit cross section corroborated the simulated temperature profile of the $14 \mathrm{~min}$ RF heating of the model fruit in $160 \mathrm{~mm}$ electrode gap (Fig. 5b).

When the model fruit was placed in the middle of $160 \mathrm{~mm}$ electrode gap, the fruit sections near to electrodes were heated at faster rate than the core of the fruit as shown in Fig 5. The uniform heating over the entire fruit section can be expected if the gap between two electrodes is very large. But, maintaining large air gap is not practical for industrial applications because a large gap reduces the electric field strength results in slow heating rates. It can be inferred from the above results that uniform RF heating of the fresh fruits would not be possible. Hallman \& Sharp (1994) also summarized the experience of many researchers and concluded that localized and uneven heating of fresh fruits is the major obstacle in using RF energy for fresh fruits.

\subsection{RF heating pattern in fruit immersed in water}

Immersion of the fresh fruits in water has been suggested by Ikediala et al.(2002) as a means to overcome the problems associated with RF heating of the fresh fruits, hence the simulation model was developed to study the effect of water on heating pattern. Fig. 6a a

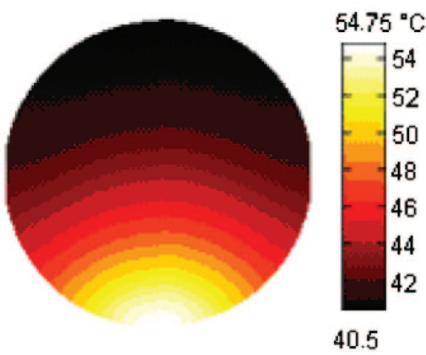

b

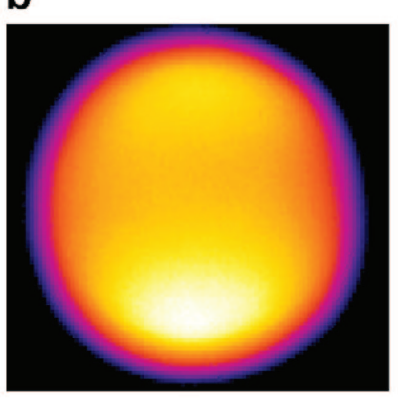

Bottom $55.8^{\circ} \mathrm{C}$

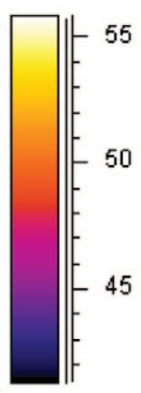

$41.3^{\circ} \mathrm{C}$
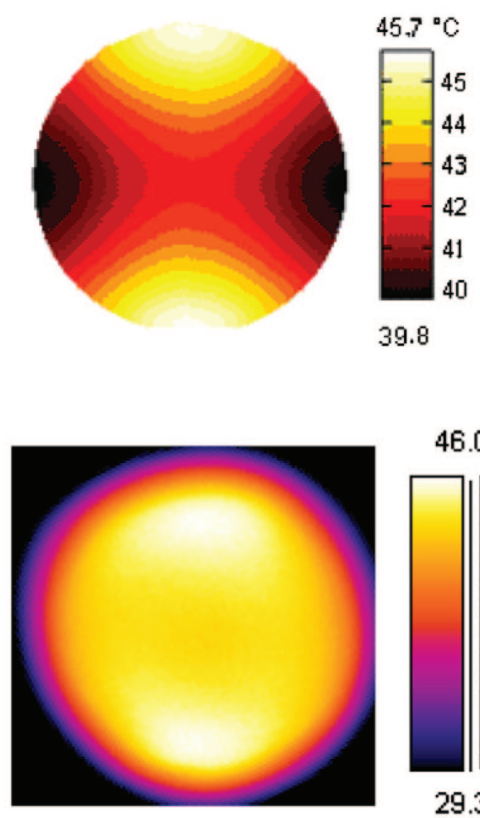

Middle
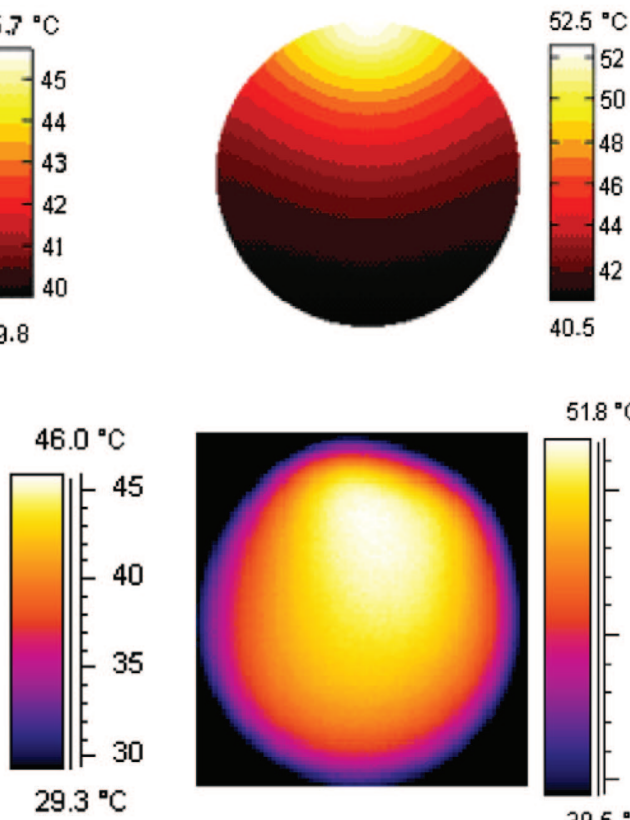

$51.8^{\circ} \mathrm{C}$

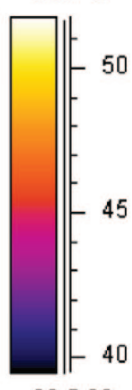

Top

Fig. 5. Simulated (a) and experimental (b) temperature distributions after 14 min of RF heating of the model fruit ( $\Phi 80$ mm) placed at $25 \mathrm{~mm}$ above the ground electrode, middle, $25 \mathrm{~mm}$ below the top eletrode in a $160 \mathrm{~mm}$ electrode gap. 
a

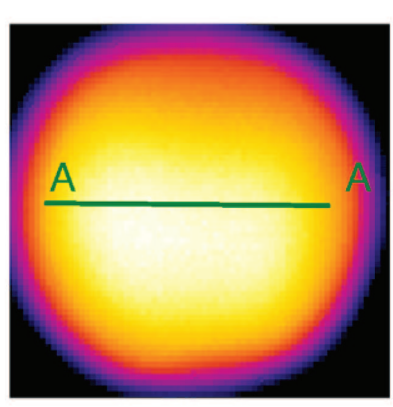

b

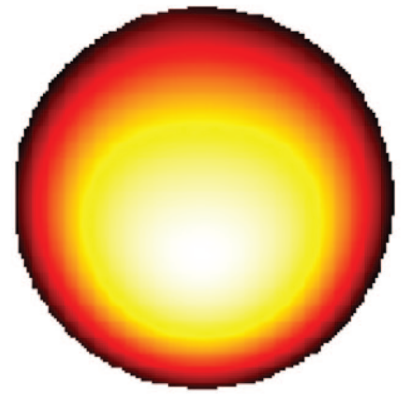

C

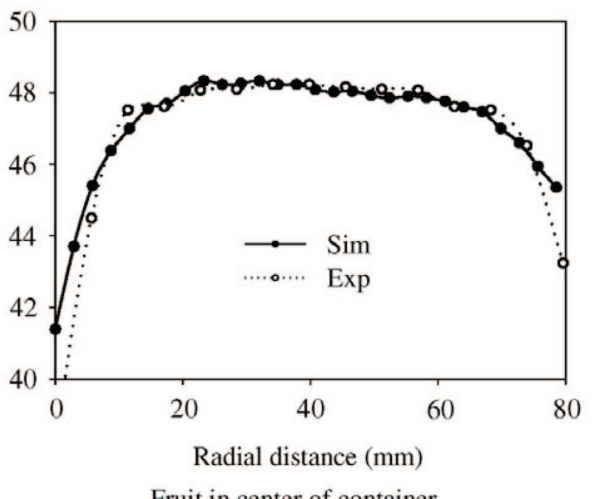

Fruit in center of container $64.8^{\circ} \mathrm{C}$

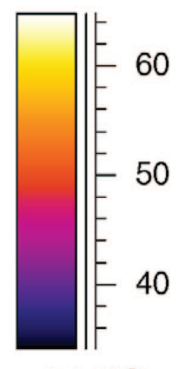

$34.1^{\circ} \mathrm{C}$

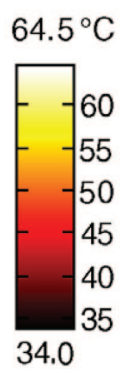

34.0

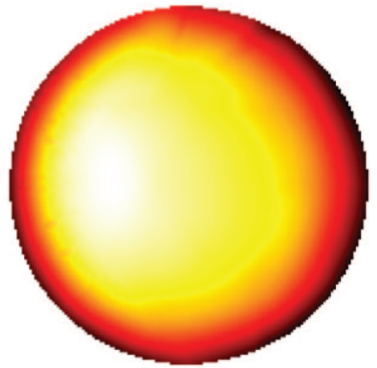

$48.4^{\circ} \mathrm{C}$
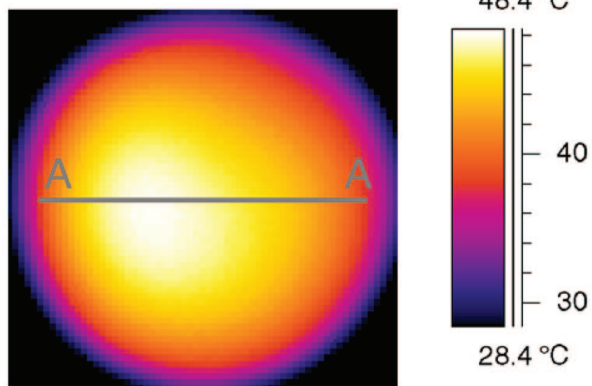

$48.0^{\circ} \mathrm{C}$

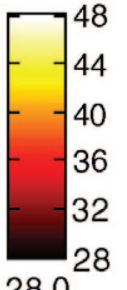

28.0

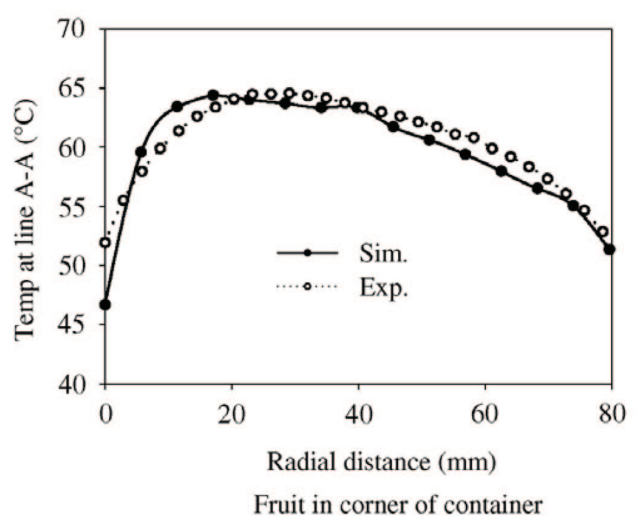

Fig. 6. Experimental (a) and simulated (b) temperature distributions inside model fruit ( $\Phi 80$ mm) and at horizontal radial line A-A (c) after 7 min of RF heating in a 195 mm electrode gap.

shows that immersion of the center-placed model fruit in water slightly shifted the hot spot toward the core of the model fruit. Moreover, presence of water radically enhanced power coupling as it took only half time (7 min) what required for RF heating of the fruits in air to reach $\sim 50^{\circ} \mathrm{C}$ temperature. The increased heating rate was attributed by lossy nature of the water which offers less resistance to electric field compared to the air.

When the model fruit moved closer to the container wall from the center of the container, maximum heating occurred at the fruit section segment close to the container wall as shown in Fig 6b. Since water provides the path of least resistance, electric fields converge at the top of the water-wall interface. The very high electric field concentration at edges passes through the model fruit having large loss factor.

The temperature profile along the line A-A as shown in Fig. 6c corroborated that simulation and experimental results agreed. Root mean square (RMS) of temperature difference between the experimental and simulated distributions at line A-A was calculated and the RMS values were $0.98^{\circ} \mathrm{C}$ and $0.74^{\circ} \mathrm{C}$ for corner and centrally placed model fruits, respectively. It was also corroborated by the experimental and simulated time-temperature history (RMS value was $1.1^{\circ} \mathrm{C}$ ) at the core of the centrally placed gel ball as shown in Fig. 7 .

\subsection{Effect of fruit rotation}

It was clear from above discussion that immersion technique alone did not completely eliminate the uneven RF heating of large fruits such as apple and orange. To overcome this problem, Birla et al. (2004) suggested to move and rotate fruits in water during RF heating. Fig. 8 shows the effect of rotation and movement of a fruit-water mix on the heating pattern of model fruit. The concentric heating pattern over the fruit cross section was observed in both experimental and simulation 


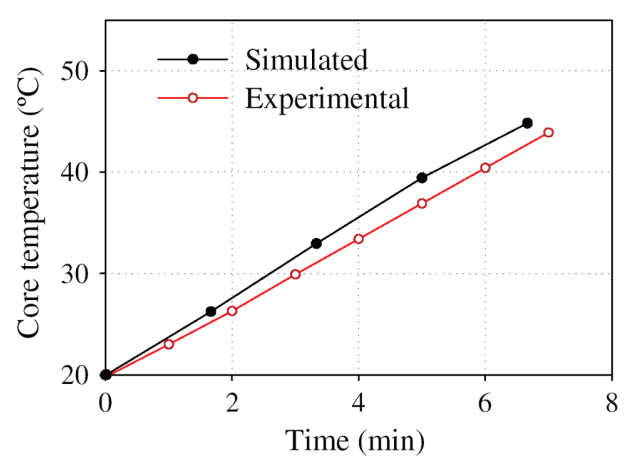

Fig. 7. Simulated and experimental time-temperature histories at the core of the centrally placed model fruit ( $\Phi 80$ $\mathrm{mm}$ ) in a water filled container after $7 \mathrm{~min}$ of RF heating in a $195 \mathrm{~mm}$ electrode gap.

temperature profiles. However, there was a discrepancy in simulated and experimental temperatures over the cross section of the model fruit. In the simulation, spherical ball was kept in the center to avail the benefits of axial symmetry, whereas in the experiment model fruit moved along the peripheral of the rectangular fruit mover. This should explain the observed discrepancy in the final temperature. It is quite logical that the rotation of the spherical objects during RF heating evened out the effect of proximity of the sphere to electrodes and container walls. It should be noted that rotation and movement of fruit did not guarantee uniform heating all over the cross section of the homogenous fruit mass because of the difference in dielectric properties of the medium and fruit. A saline water immersion technique or differential startup temperature should be adopted along with fruit movement and rotation during RF heating to minimize differential heating between fruit and water.

\subsection{Effect of dielectric properties of medium and the model fruit}

Upon validating the simulation model, the developed model was used to study the effects of dielectric properties of the model fruit and medium. One model fruit was placed in the center of the water filled container at $25 \mathrm{~mm}$ above the bottom of the electrode. The value of loss factor was varied intentionally by changing the constant $(\mathrm{C})$ in the regression Eqs. 8 and 9 such that the initial value at $20^{\circ} \mathrm{C}$ were $20,40,80$ for water and $80,160,240,300$ for the model fruit. It was assumed here that in 20 to $50{ }^{\circ} \mathrm{C}$ temperature range, the loss factor of the water or the gel varies linearly and the effect of the temperature remains unchanged. It was also assumed that the regression Eqs. for the dielectric constant remains unchanged irrespective of the intentional increase in the loss factor value. The regression Eqs. for the dielectric constant of the model fruit and the water used in this study are listed in Table 1.

$$
\begin{gathered}
\varepsilon_{w}^{\prime \prime}=0.33 T+\mathrm{C} \\
\varepsilon_{m}^{\prime \prime}=4.36 T+\mathrm{C}
\end{gathered}
$$

\subsubsection{Loss factor $\left(\boldsymbol{\varepsilon}^{\prime \prime}\right)$ of water}

Fig. 9a shows the influence of increasing loss factor of medium on the temperature profile at the vertical line drawn through the center of the model fruit placed in centre of $25 \mathrm{~mm}$ above the bottom of the container. An increase in the water loss factor (by increasing ' $\mathrm{C}$ ' value in Eq. 8, and using all other properties of the fruit and the water as listed in Table 1) reduced the peak temperature inside the fruit, as saline water was likely to provide a conductive media for EM energy to pass through water as the path of least resistance.

Fig. $9 \mathrm{~b}$ summarizes the results of a series of simulations with water loss factor initial values at $20^{\circ} \mathrm{C}$ ranging from 20 to 180. Increasing water loss factor increased the water temperature but the temperature started to decline after a certain water $\varepsilon^{\prime \prime}$ value $(\sim 80)$. Altering the dielectric properties of water by addition of salt minimizes the differential heating between the fruit (oranges and cherries) and water (Birla et al., 2004; Ikediala et al., 2002). Differential heating between fruit and water can be minimized by appropriately matching dielectric properties of the water with that of fruit.

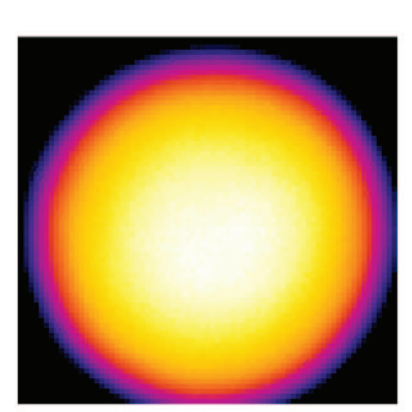

Experimental

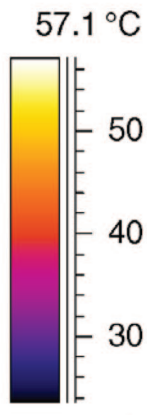

$23.6^{\circ} \mathrm{C}$

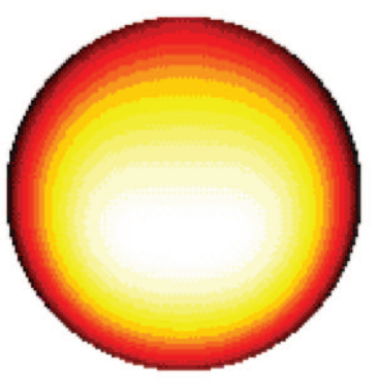

$52.5^{\circ} \mathrm{C}$

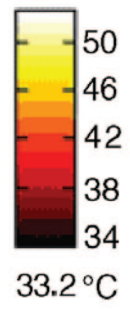

Simulated

Fig. 8. Experimental and simulated temperature distributions of model fruits $(\Phi 80 \mathrm{~mm})$ rotating and moving in the fruit mover (Birla et al., 2006) after 6 min of RF heating in a $195 \mathrm{~mm}$ electrode gap. 
(a) Temperature profile along the container height

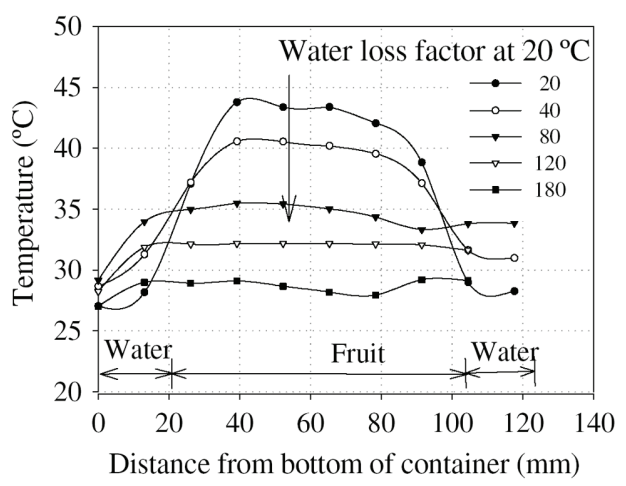

(b) Temperature at fruit core and water surface

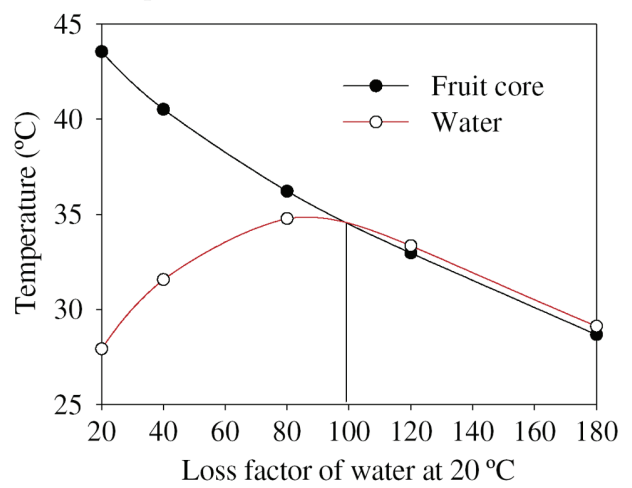

Fig. 9. Simulated effect of the water loss factor on heating patterns inside the model fruit $(\Phi 80 \mathrm{~mm})$ placed in the center and at $25 \mathrm{~mm}$ above the bottom of the container and subjected to $7 \mathrm{~min}$ RF heating in a $195 \mathrm{~mm}$ electrode gap.

\subsubsection{Loss factor of fruit}

Fig. 10 shows the simulated effect of the increasing fruit loss factor on the temperature profile at the vertical line drawn through the center of the model fruit placed in centre of $25 \mathrm{~mm}$ above the bottom of the container $(7$ min of RF heating with a $195 \mathrm{~mm}$ electrodes gap). The simulation results showed that increasing the $\varepsilon^{\prime \prime}$ value of the model fruit (by increasing the constant ' $\mathrm{C}$ ' in the Eq. 9 , and using all other properties of the tap water and the model fruit as listed in Table 1) the resulted in increasing heating rate only to a certain value $\left(\varepsilon^{\prime \prime}=180\right)$, which was contrary to a general belief that increasing loss factor constantly increases heating rate inside the fruit. But beyond that value increasing loss factor diminished the heating rate inside the fruit. This can be explained by the fact that power density is proportional directly not only to the loss factor, but also to the square of electric field intensity. Increasing loss factor decreases the electric field intensity hence maximum heating rate occurs at particular loss factor value. An expression for limiting $\varepsilon^{\prime \prime}$ value as a function of the dielectric properties of a surrounding medium was derived by Birla (2006). Now with this simulation model and analytical expression a faster heating of apple $\left(\varepsilon^{\prime \prime}=120\right)$ in comparison with oranges $\left(\varepsilon^{\prime \prime}=220\right)$ can be explained. (a) Temperature at vertical line dawn from the fruit core

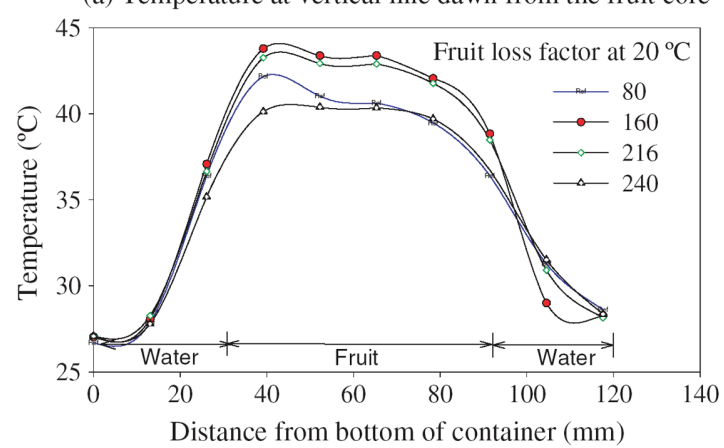

(b) Temperature at fruit core

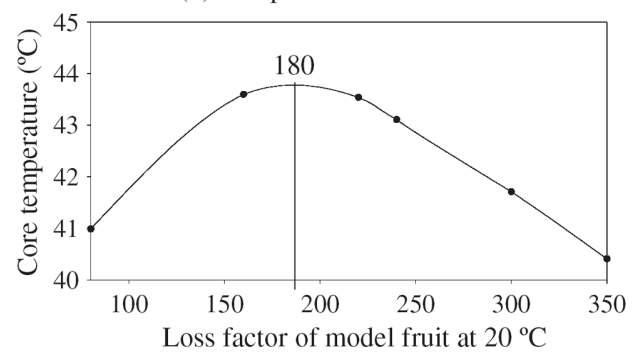

Fig. 10. Simulated effects of the dielectric loss factor of the model fruit on the temperature distribution inside the fruit $(\Phi 80 \mathrm{~mm})$ placed in the center and at $25 \mathrm{~mm}$ above the bottom of the water filled container and subjected to $7 \mathrm{~min}$ RF heating in a $195 \mathrm{~mm}$ electrode gap.

\subsection{Limitation of the model}

The computer model developed in this study was able to predict the transient temperature profiles using physical and temperature-dependent dielectric properties, which are needed to design a RF heating process for disinfection of fruits. Having predicted the timetemperature profile in large fruit, one can decide the adequacy of the heating process based on information about the thermal death kinetics of insects/pests and quality degradation kinetics. In the present model, we assumed constant voltage over the entire upper electrode. However, in practice, this would not be true due to the effects of distributed system parameters and high frequency. To take these factors into account for the proper design of a whole RF system, an EM wave equation should be solved for domains including RF generator, tank circuit and applicator.

\section{Conclusions}

A computer model based on finite element method was developed to the quasi-static electric fields in an RF heating system. The coupled Maxwell's EM equations and Navier-Stokes equations were solved for a 3-D model using FEMLAB software. The simulation results were validated with experimental temperature profiles of gellan gel model fruit. The experimental and simulation results were found to be in agreement, as the model 
was able to predict transient temperature profiles using physical and temperature-dependent dielectric properties. The validated computer model was further used to study the effects of dielectric properties of the medium and fruits, and effect of fruits rotation. Non-uniform heating is attributed to shape, dielectric properties, and relative fruit position in the container. The predicted temperature profile may be useful for designing an RF heating process in disinfestation of fruits. The developed model can be used as a tool to study the heating pattern of various fruits as influenced by dielectric properties of peel, pulp, and inner core.

Acknowledgment - This research was supported by Washington State University (WSU) Agricultural Research Center and WSU Impact Centre. The project was also additionally supported by grants from BARD (US-3276-01) and USDA-NRI (2005-35503-16223). We acknowledge the support of COMSOL technical team for their technical assistance on FEM model development. We thank Dr. Slava Komrov for suggestions and feedbacks on the model.

\section{References}

Birla, S. L. (2006). Potential of radio frequency heating of fresh fruits as an alternative quarantine method. PhD dissertation: Washington State University, Biological Systems Engineering.

Birla, S. L., Wang, S., \& Tang, J. (2006). Radio frequency heating patterns as influenced by dielectric properties of constituent parts of fruits. In: Proc. ASABE, Paper no. 066052. eds. Portland: ASABE.

Birla, S. L., Wang, S., Tang, J., \& Hallman, G. (2004). Improving heating uniformity of fresh fruit in radio frequency treatments for pest control. Postharvest Biology and Technology, 33, 205-217.

Chan, T. V. C. T., Tang, J., \& Younce, F. (2004). 3-Dienstional numerical modeling of an industrial Radio frequency heating systems using Finite elements. Journal of Microwave Power and Electromagnetic Energy, 39(2), 87-105.

Choi, C. T. M., \& Konrad, A. (1991). Finite-Element modeling of the RF heating process. IEEE Transactions on Magnetics, 27(5), 4227-4230.

COMSOL (2006). FEMLAB 3.3 User Guide, Comsol Multiphysics, Los Angeles.

Frings, H. (1952). Factors determining the effects of radio-frequency electromagnetic fields and materials they infest. Journal of Economic Entomology 45, 396-408.

Guan, D., Cheng, M., Wang, Y., \& Tang, J. (2004). Dielectric properties of mashed potatoes relevant to microwave and radiofrequency pasteurization and sterilization processes. Journal of Food Science, 69(1), FEP30-FEP37.

Hallman, G. J., \& Sharp, J. L. (1994). Radio frequency heat treatments. In Quarantine treatments for pests of food plants, 165-170. Westview Press, San Francisco, CA.

Ikediala, J. N., Hansen, J. D., Tang, J., Drake, S. R., \& Wang, S. (2002). Development of a saline water immersion technique with RF energy as a postharvest treatment against coding moth in cherries. Postharvest Biology and Technology, 24, 25-37.

Marra, F., Lyng, J., Romano, V., \& McKenna, B. (2007). Radiofrequency heating of foodstuff: Solution and validation of a mathematical model. Journal of Food Engineering, 79(3), 998-1006.
Marshall, M. G., \& Metaxas, A. C. (1998). Modeling of the radio frequency electric field strength developed during the RF assisted heat pump drying of particulates. Journal of Microwave Power and Electromagnetic Energy, 33(3), 167-177.

Metaxas, A. C. (1996). Foundations of Electoheat - A Unified Approach, Ist ed., John Wiley \& Sons, New York.

Nelson, S. O., \& Payne, J. A. (1982). RF dielectric heating for pecan weevil control. Transactions of ASAE, 31, 456-458.

Neophytou, R. I., \& Metaxas, A. C. (1996). Computer simulation of a radio frequency industrial system. Journal of Microwave Power and Electromagnetic Energy, 31(4), 251-259.

Neophytou, R. I., \& Metaxas, A. C. (1997). Characterisation of radio frequency heating systems in industry using a network analyser. IEE Proceedings-Science Measurement and Technology, 144(5), 215-222.

Neophytou, R. I., \& Metaxas, A. C. (1998). Combined 3D FE and circuit modeling of radio frequency heating systems. Journal of Microwave Power and Electromagnetic Energy, 33(4), 243-262.

Neophytou, R. I., \& Metaxas, A. C. (1999). Combined tank and applicator design of radio frequency heating systems. IEE Proceedings-Microwaves Antennas and Propagation, 146(5), 311-318.

Rehman, S. (1995). Food Properties Handbook, CRC Press, New York.

Stogryn, A. (1971). Equations for calculating the dielectric constant of saline water. IEEE Transactions of Microwave Theory Technique, MTT-19, 733-736.

Tang, J., Ikediala, J. N., Wang, S., Hansen, J. D., \& Cavalieri, R. P. (2000). High-temperature-short-time thermal quarantine methods. Postharvest Biology and Technology, 21(1), 129-145.

Tang, J., Tung, M. A., \& Zeng, Y. (1995). Mechanical properties of gellan gels in relation to divalent cations. Journal of Food Science, 60(4), 748-752.

Tang, J., Tung, M. A., \& Zeng, Y. (1997). Gelling properties of gellan solutions containing monovalent and divalent cations. Journal of Food Science, 62(4), 688-692, 712.

Veen, M. E. v. d., Goot, A. J. v. d., Vriezinga, C. A., Meester, J. W. G. d., \& Boom, R. M. (2004). On the potential of uneven heating in heterogeneous food media with dielectric heating. Journal of Food Engineering, 63(4), 403-412.

von Hippel, A. R. (1995). Dielectric Materials and Applications, Arctech House, Boston.

Wang, S., Ikediala, J. N., Tang, J., Hansen, J. D., Mitcham, E., Mao, R., \& Swanson, B. (2001). Radio frequency treatments to control codling moth in in-shell walnuts. Postharvest Biology and Technology, 22(1), 29-38.

Wang, S., Tang, J., \& Cavalieri, R. P. (2001). Modeling fruit internal heating rates for hot air and hot water treatments. Postharvest Biology and Technology, 22(3), 257-270.

Wang, S., Tang, J., Cavalieri, R. P., \& Davis, D. C. (2003a). Differential heating of insects in dried nuts and fruits associated with radio frequency and microwave treatments. Transactions of the ASAE, 46(4), 1175-1182.

Wang, S., Tang, J., Johnson, J. A., Mitcham, E., Hansen, J. D., Cavalieri, R. P., Bower, J., \& Biasi, B. (2002). Process protocols based on radio frequency energy to control field and storage pests in in-shell walnuts. Postharvest Biology and Technology, 26(3), 265-273.

Wang, S., Tang, J., Johnson, J. A., Mitcham, E., Hansen, J. D., Hallman, G., Drake, S. R., \& Wang, Y. (2003b). Dielectric properties of fruits and insect pests as related to radio frequency and microwave treatments. Biosystems Engineering, 85(2), 201-212.

Yang, J., Zhao, Y., \& Wells, J. H. (2003). Computer simulation of capacitive radio frequency (RF) dielectric heating on vegetable sprout seeds. Journal of Food Process Engineering, 26(3), 239-263.

Zhang, Q., Jackson, T., \& Ungan, A. (2000). Numerical modeling of microwave induced natural convection. International Journal of Heat and Mass Transfer, 43, 2141-2154. 